\title{
Atrial fibrillation detection using support vector machine and electrocardiographic descriptive statistics
}

\section{Nuryani Nuryani*, Bambang Harjito, Iwan Yahya and Maratus Solikhah}

Faculty of Mathematics and Natural Sciences,

University of Sebelas Maret,

Surakarta 57126, Indonesia

Email: nuryani@mipa.uns.ac.id

Email: bambang_harjito@staff.uns.ac.id

Email: iyahya@mipa.uns.ac.id

Email: s.maratus@gmail.com

*Corresponding author

\section{Rifai Chai}

Centre for Health Technologies,

University of Technology,

Sydney, Australia

Email: Rifai.Chai@uts.edu.au

\author{
Anik Lestari \\ Faculty of Medicine, \\ University of Sebelas Maret, \\ Surakarta 57126, Indonesia \\ Email: leslietarinick@yahoo.com
}

\begin{abstract}
This paper proposes a new technique for detecting atrial fibrillation (AF). The method employs electrocardiographic features and support vector machine (SVM). The features include descriptive statistics of electrocardiographic RR interval. The RR interval is the distance in time between two consecutive R-peaks of electrocardiogram. AF detections using SVM with different electrocardiographic features and different SVM free parameters are explored. Employing SVM with the optimal free parameters and all the proposed electrocardiographic features, we find an AF detection technique with a comparable performance. The best performance obtained by the technique is $98.47 \%$ and $97.84 \%$, in terms of sensitivity and specificity.
\end{abstract}

Keywords: atrial fibrillation; support vector machine; electrocardiogram; RR interval. 
Reference to this paper should be made as follows: Nuryani, N., Harjito, B., Yahya, I., Solikhah, M., Chai, R. and Lestari, A. (2017) 'Atrial fibrillation detection using support vector machine and electrocardiographic descriptive statistics', Int. J. Biomedical Engineering and Technology, Vol. 24, No. 3, pp.225-236.

Biographical notes: Nuryani Nuryani is with Department of Physics, University of Sebelas Maret, Surakarta, Indonesia. He completed his Doctoral Degree in the Centre for Health Technologies, University of Technology Sydney, Australia. His current research interests include electrocardiography, support vector machine and computational intelligence for biomedical signal processing.

Bambang Harjito is with Department of Informatics, University of Sebelas Maret, Surakarta, Indonesia. He completed his doctoral degree in School of Information System, Curtin University, Perth, Australia in 2013. He leads the information data security research group at Computer Science Department FMIPA UNS targeting issues in data protection and privacy protection, information hiding, cryptography, watermarking and data protection in wireless sensor networks.

Iwan Yahya is a Senior Lecturer at Physics Department, Sebelas Maret University. His research interest is acoustics, speech analysis and digital signal processing. He is currently in charge of Acoustics Research Laboratory at Faculty of Science, Sebelas Maret University.

Maratus Solikhah is with Department of Physics, University of Sebelas Maret, Surakarta, Indonesia. His current research includes electrocardiography, heart arrhythmia detection and neural network.

Rifai Chai received his PhD in Engineering from the University of Technology, Sydney (UTS). Currently, he is an Associate Lecturer in the Centre for Health Technologies, Faculty of Engineering and Information Technology at the UTS. His current research interests on brain-computer interfaces, biomedical instrumentation, biomedical signal processing computational intelligence using neural networks, fuzzy logic, and evolutionary computation and embedded system design.

Anik Lestari is with Department of Public Health, Medical Faculty, University of Sebelas Maret, Surakarta, Indonesia. She completed her master degree and medical doctor from the same university. Her current research interests include electrocardiography and family medicine.

\section{Introduction}

Atrial fibrillation (AF) is a serious heart problem originated from the upper chamber of the heart, namely the atrial. During AF the atrial beats chaotically and irregularly. The prevalence of AF is estimated to 2.3 million in the USA and 4.5 million in the Europe (Lainscak et al., 2008). A patient with AF has risk of stroke (Healey et al., 2012) which leads to frequent hospitalisation and increases mortality (Chugh et al., 2014).

Studies have been conducted to propose methods for AF early detection. Early information of AF presence in a patient is essential to perform a quick appropriate action. 
This action could prevent the serious complications associated with AF. Varied algorithms and the associated electrocardiographic features have been studied to find a sophisticated AF detection system. The system is dedicated to detect AF accurately and quickly.

A neural network algorithm has been studied for AF detection by Daqrouq et al. (2014). The algorithm employs probabilistic neural network and utilises a wavelet feature of electrocardiogram. A different strategy of an AF detection using neural network algorithm is proposed by Mateo and Rieta (2013). This algorithm uses Radial Basis Function (RBF) neural network and utilises electrocardiographic features by cancelling the electrocardiographic QRST complex.

In addition, studies have explored a Support Vector Machine (SVM) for AF detection. SVM is a reliable classification method and has been proved to provide good performances in various medical diagnostics (Nuryani et al., 2012; Saini et al., 2014; Suchetha et al., 2013). In varied applications, SVM outperforms other algorithms (Foley et al., 2012; Saleh et al., 2011). The most recent AF detection method which utilises SVM is presented by Asgari et al. (2015). The method employs SVM which utilises RBF with the free RBF parameter $\gamma=1$. The method also employs a stationary wavelet transform to find the appropriate electrocardiographic features. The other SVM method for AF detection is proposed by Mohebbi and Ghassemian (2008). The method employs a Linear Discriminant Analysis (LDA) for feature reduction which is used for the input of SVM. Moreover, Colloca et al. (2013) study SVM for AF detection by comparing electrocardiographic R-peaks-related features.

The aim of this paper is to explore SVM more widely for AF detection. So far, in the previous studies for atrial fibrillation, SVM is set with a single value of SVM free parameter, or another study, the employed parameter value is not clearly mentioned (Asgari et al., 2015; Colloca et al., 2013). The SVM free parameter is commonly called as $C$ parameter. Studies have shown that the appropriate $C$ parameter could provide good performance (Nuryani et al., 2014; Preetha and Suresh, 2014).

Furthermore, in AF detection studies, variation of the free kernel function parameters is not widely explored yet, whereas utilising suitable values of the free parameters could provide good performance of a detection (Li et al., 2015). In SVM technique, a kernel function is used for the mapping input data to higher dimension. Thus, this paper presents SVM for AF detection with variation of free parameters of SVM and its kernel function.

In addition, this paper also presents the descriptive statistics features of electrocardiographic RR intervals as the input of SVM in AF detection. So far, the descriptive statistics features of RR interval are not yet intensively evaluated. Electrocardiographic RR interval is chosen as it is strong indicator of AF (Lian et al., 2011). Furthermore, this paper compares the window or segment length, as other studies conduct (Larburu et al., 2011).

The rest of this paper is organised as follows. The second section describes the method providing features extraction and SVM design. The experimental result is presented in the third section. The fourth section presents the discussion. Finally, the conclusion is provided in the last section. 


\section{Method}

In general, the detection strategy is presented in Figure 1. RR intervals, the electrocardiographic features used in this study, are obtained to find their descriptive statistics. These features are used for the inputs of SVM. The output of SVM is the decision, namely AF or non-AF.

Figure 1 A detection strategy is to detect atrial fibrillation

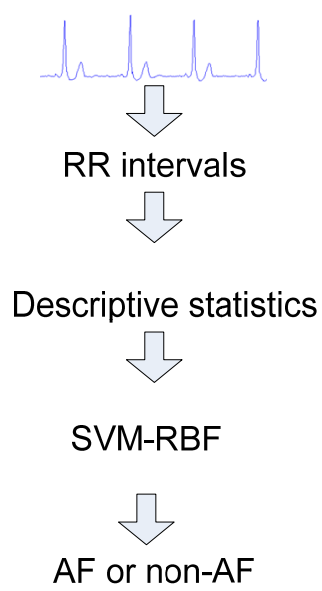

\subsection{Electrocardiographic features}

To find RR intervals, we use the QRS peaks of patient's electrocardiogram from MITBIH AF database (Goldberger et al., 2000). The RR interval is the difference in time between two consecutive QRS peaks. The descriptive statistics of RR intervals are obtained using the following steps. Firstly, a QRS-peaks series is segmented. The length of each segment is along $n$ QRS peaks. Three different $n$ are investigated, i.e. 20, 40 and 60. Secondly, descriptive statistics of the individual segment are calculated. The descriptive statistics involve 26 types as presented in Table 1 . The statistics consist of two groups, namely a group related to RR interval and another group related to the difference between two consecutive RR intervals (dRR). Each group consist of 13 statistics. The detail of the statistics is presented in Table 1. Thirdly, each segment is defined as AF or non-AF. The segment is defined as AF if the number of AF beats in the segment is more than $\alpha$. Three different $\alpha$ are evaluated, namely $30 \%, 50 \%$ and $70 \%$. Thus, in each segment type, we have 26 features and the associated AF or non-AF label.

\subsection{SVM classification}

The detail of SVM classification could be found in the paper presented by Burges (1998). Basically, an SVM classification constructs an optimal hyper-plane which separates two classes. Let $X=\left(\mathbf{x}_{i}, z_{i}\right)$ is a training data set which can be linearly separated. $\mathbf{x}_{i} \in R^{n}$ is data of $n$ dimensional space with the associated class label $z_{i} \in[-11] . I=1,2, \ldots, n$, with $n$ is the number of data. The optimal hyper-plane separating a two-class training 
data maximally is defined by $\mathbf{w} \cdot \mathbf{x}+b=0$, where $\mathbf{w}$ is the hyper-plane perpendicular vector. The training data satisfying $z_{i}(\mathbf{w} \cdot \mathbf{x}+b)-1 \geq 0$ which lay in the equality are called as support vectors. The optimum hyper-plane can be found by maximising distance between two hyper-planes: $\mathbf{w} \cdot \mathbf{x}+b=+1$ and $\mathbf{w} \cdot \mathbf{x}+b=-1$. The distance between those two hyper-planes is $2 /\|\mathbf{w}\|$.

Table 1 Descriptive statistics of RR intervals for electrocardiographic features

\begin{tabular}{clcl}
\hline No & & No & \\
\hline & RR-related features & & dRR-related features \\
\hline 1 & Standard deviation of RR & 1 & Standard deviation of dRR \\
2 & Average of RR & 2 & Average of dRR \\
3 & Range of RR & 3 & Range of dRR \\
4 & Geometric mean of RR & 4 & Geometric mean of dRR \\
5 & Harmonic mean of RR & 5 & Harmonic mean of dRR \\
6 & Interquartile range of RR & 6 & Interquartile range of dRR \\
7 & Minimum of RR & 7 & Minimum of dRR \\
8 & Trimmed mean of RR & 8 & Trimmed mean of dRR \\
9 & Kurtosis of RR & 9 & Kurtosis of dRR \\
10 & Skewness of RR & 10 & Skewness of dRR \\
11 & Maximum of RR & 11 & Maximum of dRR \\
12 & Median of RR & 12 & Median of dRR \\
13 & Mode of RR & 13 & Mode of dRR \\
\hline
\end{tabular}

For real data of world problems, such separating hyper-plane mostly does not exist. Therefore, a slack variable $\xi_{i}$ is introduced and hence $\mathrm{z}_{i}(\mathbf{w} \cdot \mathbf{x}+b) \geq 1-\xi_{\mathrm{i}}$. The optimal separating hyper-plane is determined by minimising:

$$
C \sum_{i=1}^{k} \xi_{i}+\frac{1}{2}\|\mathbf{w}\|^{2}
$$

where $C$ is a cost constant that is used to control the trade-off between margin size and error.

Finding the optimal hyper-plane is conducted by using Lagrange multiplier approach through the maximisation of subject to:

$$
\begin{aligned}
& L(\alpha)=\sum_{i=1}^{n} \alpha_{i}-\frac{1}{2} \sum_{i=1}^{n} \sum_{j=1}^{n} \alpha_{i} \alpha_{j} z_{i} z_{j}\left(\mathbf{x}_{i} \cdot \mathbf{x}_{j}\right) \\
& 0 \leq \alpha_{i} \leq C \\
& \sum_{i=1}^{n} z_{i} \alpha_{i}
\end{aligned}
$$

where $\alpha_{i}$ is the Lagrange multiplier.

The term in the parentheses in equation (2) is then be replaced by a kernel function $K\left(\mathbf{x}_{i}, \mathbf{x}_{j}\right)$ to map data to a higher dimensional space. By this mapping, non-linearly 
separable data could be linearly classified. In this paper, RBF function is employed for the mapping. The formulation of the RBF function is:

$$
k\left(x_{i}, x_{j}\right)=\exp \left(-y\left\|x_{i}-x_{j}\right\|^{2}\right)
$$

In this paper, difference values of $C$ in equation (3) and $\gamma$ in equation (4) are tested.

Finally, the class prediction for any test data $\mathbf{x}_{i} \in R^{n}$ is given by:

$$
\mathrm{f}(\mathbf{x})=\left(\sum \alpha_{i} y_{i} k\left(\mathbf{x}_{i}, \mathbf{x}\right)+b\right)
$$

The value of $f(\mathbf{x})$ which is greater than 0 is associated with AFib class (AFib class) and the negative one is associated with non-AFib.

\section{Results}

This paper presents a study of atrial fibrillation detection techniques. Two main objectives are studied: exploring descriptive statistics features of RR intervals and exploring SVM designs in relation with AF detection. These features are for the inputs of SVM.

Firstly, the detection performances with different inputs are presented in Figure 2. The inputs are the statistics of RR interval. This performance is obtained by the SVM with $C=10$ and $\gamma=1$. The performance with one input, two inputs, three inputs, and so forth is presented. The order of the input is randomly selected. It means that which inputs used as the first input, the second input, and so on are selected randomly. The result shows that each input could positively contribute to the detection performance. The accuracy does not significantly increase with the input addition when the inputs number is around 8 . The highest performance of this approach is $96.98 \%$ in terms of accuracy.

Figure 2 Performance of the SVM AF detection using descriptive statistics of RR intervals

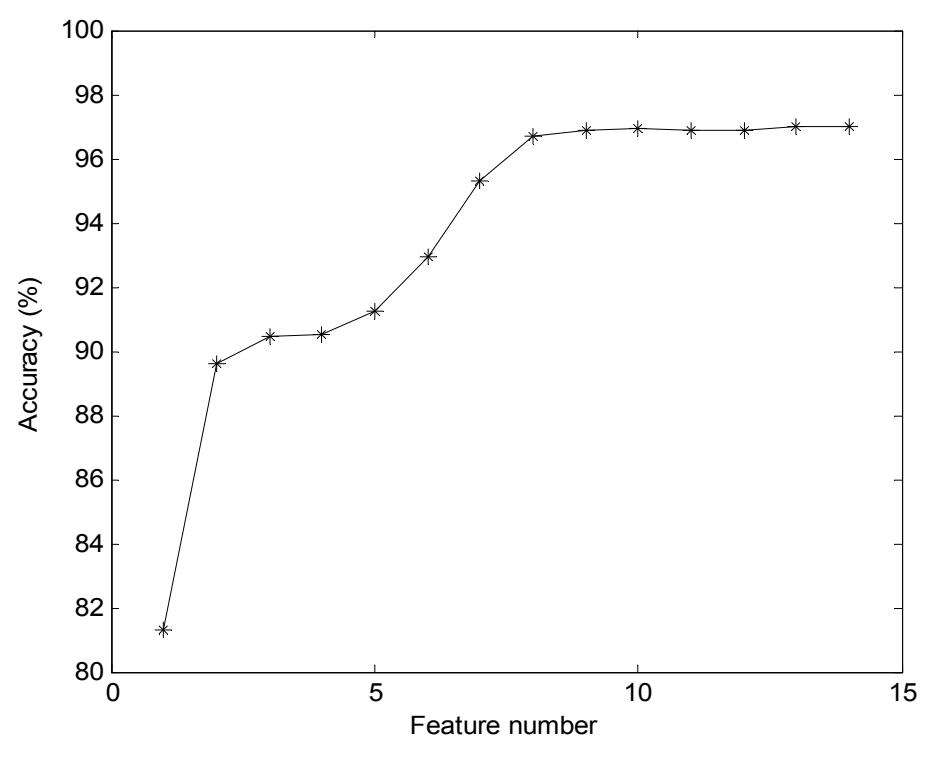


Secondly, the performance of the detection employing the statistics of dRR is presented in Figure 3. As the previous one, the order of the input is randomly selected and the value of $C$ and $\gamma$ of SVM is set to 10 and 1 . The best performance of this approach is $93.54 \%$ in terms of accuracy. The performance of the SVM does not increase significantly when the input number has reached 3 . Thirdly, all the 26 inputs are employed. The inputs consist of both the statistics of RR and dRR. The result is presented in Figure 4. In general, the performance increases with the input number addition. The best performance found by this approach is $97.64 \%$ which is higher than the two previous approaches. The previous approaches employ either RR statistics only or dRR statistics only.

Figure 3 Performance of the SVM AF detection using descriptive statistics of RR and dRR intervals

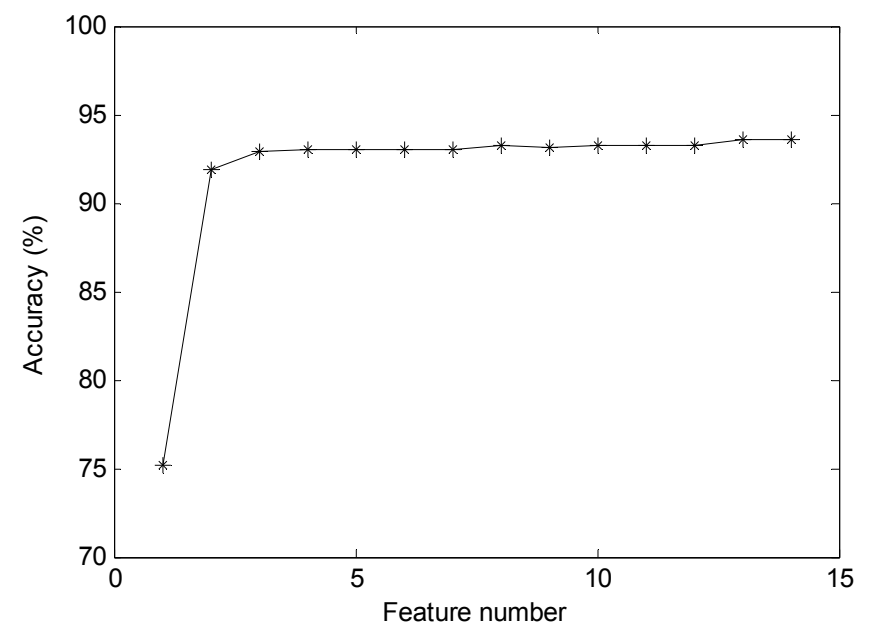

Figure 4 Performance of the SVM AF detection using descriptive statistics of RR and dRR intervals

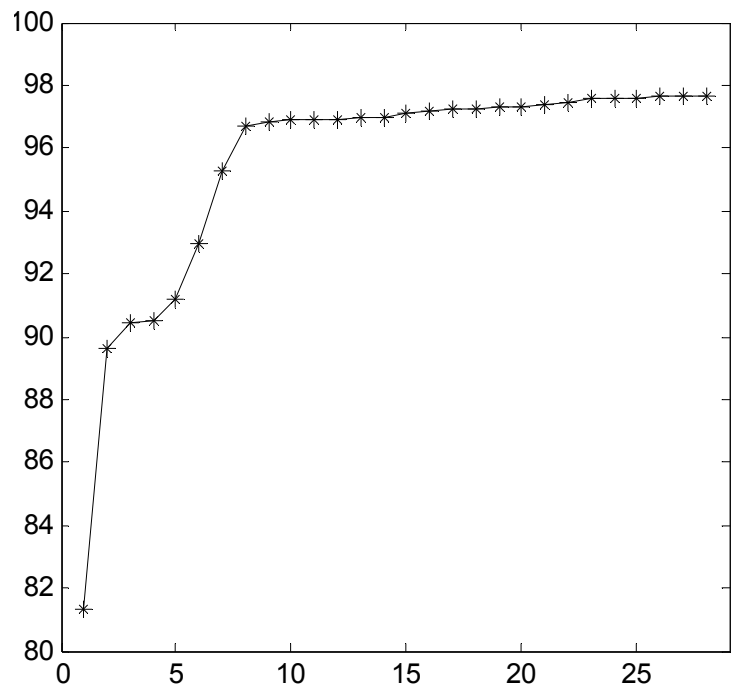


Figure 5 Performance of the SVM AF detection varying the $C$ and $\gamma$ value

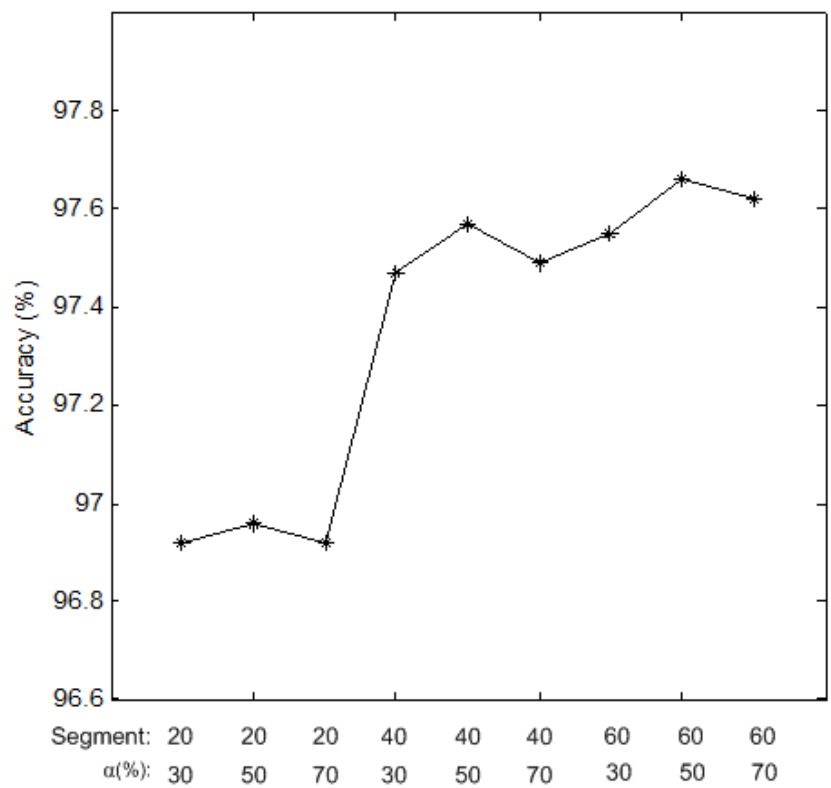

Fourthly, all 26 statistic features are employed with varying the segment length and the $\alpha$ value. Three types of segment length are tested. The three length types are 20, 40 and 60 heart beats. In addition, three $\alpha$ values $(30 \%, 50 \%$ and $70 \%)$ are evaluated for each segment. As mentioned above, $\alpha$ is a threshold to define that a segment is belong to $\mathrm{AF}$ or non-AF. Thus, the three segment types and three $\alpha$ values generate nine combinations of features. The result of the fourth approach is presented in Figure 5. The figure shows that $\alpha=50 \%$ is producing the highest performance consistently rather than the other two alphas in every type of segment length. Besides, the feature with the segment length of 60 yields the highest performance among the other two segment lengths. Thus, the detection which employs the feature with the segment length of 60 and $\alpha=50 \%$ provides the highest performance, namely $97.64 \%$, among the other eight combinations.

Finally, the final approach, we evaluate different SVMs which employ the best feature obtained in the fourth result above. The SVM uses $\gamma$ of 1, 10, 100 and 1000 and $C$ of 1, 10, 100,1000,10,000 and 100,000 (Figure 6). The result shows that using $\gamma=1000$ provides the worst performance. The higher performance is obtained when $\gamma=100$ is used. But this performance is still lower than the SVMs which use $\gamma=100$ and $\gamma=10$. The SVM performance which utilises $\gamma=10$ is the highest when $C$ is set to 1 and 10 . However, the performance using $\gamma=10$ shows the lower result rather than $\gamma=1$ if $C$ is set to $1000,10,000$ and 100,000 . Considering all the variation of $\gamma$ and $C$, the best detection performance of SVM is found when both $\gamma$ and $C$ are set to 10 with $98.37 \%$ accuracy. The associated sensitivity and specificity are $98.47 \%$ and $97.84 \%$, respectively. 
Figure 6 Performance of the SVM AF detection varying the $C$ and $\gamma$ value

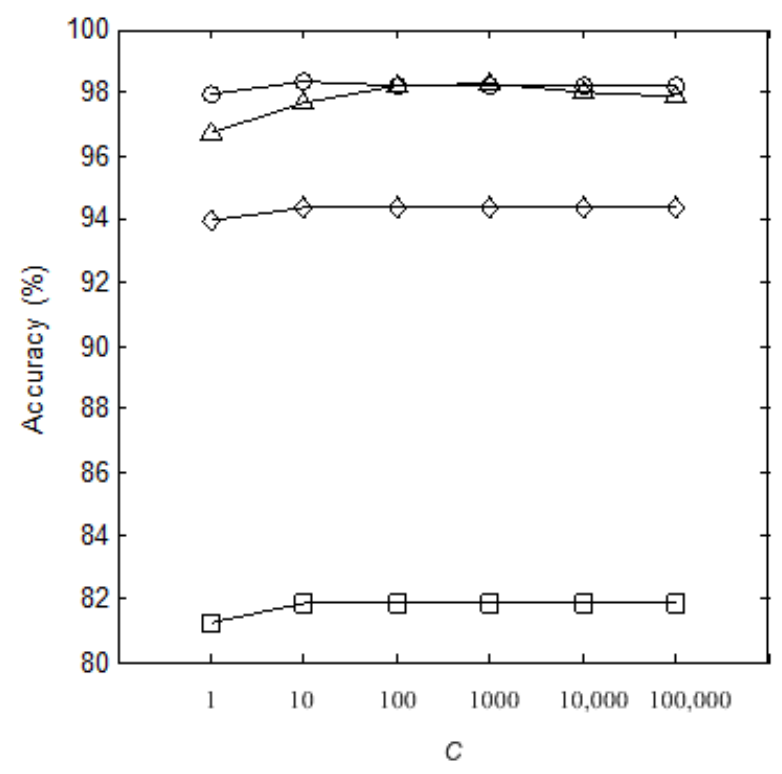

The performance obtained by this study is comparable to the previous studies. The comparison of AF detection approaches presented around the last lustrum is described in Table 2. The propose method provides higher sensitivity $(98.47 \%)$ than the other four approaches. Huang et al. (2011) provides method with higher specificity than the proposed method $(98.1 \%$ vs $97.84 \%)$, but their sensitivity is lower (96.1\%). The other three methods provide lower sensitivities.

Table 2 The comparison to the AF detection approaches presented around the last decade

\begin{tabular}{cccc}
\hline No & Algorithm & Sensitivity (\%) & Specificity (\%) \\
\hline 1 & Huang et al. (2011) & 96.1 & 98.1 \\
2 & Lee et al. (2013) & 98.2 & 97.7 \\
3 & Jiang et al. (2012) & 98.2 & 97.5 \\
4 & Asgari et al. (2015) & 97.0 & 97.1 \\
5 & Proposed method & 98.47 & 97.84 \\
\hline
\end{tabular}

\section{Discussions}

Until recently atrial fibrillation detection methods are still being studied by research groups. These methods are investigated to find a detection system which can detect AF early and accurately. The quick and accurate AF detection could lead appropriate AF management and could avoid further chronic complications.

This paper presents an AF detection strategy using descriptive statistics features of $R R$ intervals and the difference of two consecutive RR intervals (dRR) which are not widely explored yet. The studied features include the descriptive statistics of $R R$ 
and dRR. dRR as an AF indicator is introduced by Lian et al. (2011). In their study, Lian et al. (2011) use Cartesian coordinate to show RR-dRR map to indicate AF and non-AF. Thus, the statistics of $\mathrm{dRR}$ is used in this paper as addition to the statistics of RR. The experimental result shows that dRR statistics could improve the detection performance.

The result of the detection using the RR features shows that generally the first eight features show significant contribution to increase the detection performance. But the ninth feature onwards could not increase the performance significantly. Similar situation also happen when SVM uses the dRR features. The difference is that, for dRR, the features do not increase significantly the performance after the third feature. When both RR and dRR are employed, including the 26 features, the ninth feature onwards does not provide significant improvement of the performance. The best performance is obtained when all the 26 features are included.

The SVMs performances with different free parameters $C$ and $\gamma$ are presented in this paper. The presentation could provide how the parameters influence the detection performances. As presenting in Figure 6, the SVM using higher $\gamma$ provides lower performance, except when the value of $\gamma$ used is 10 which shows outstanding performance rather than $\gamma=1$ when $C=1$ and 10. The SVM performance increases by using $\gamma=10$ and varying the $C$ value from 1 to 10 . But the performance decreases by varying $C$ from 10 to 105 . The optimum is found at $\gamma=10$ and $C=10$. The optimum $C$ and $\gamma$ could be different for different real data, and thus the optimum value for a real data needs to be found. Using the work provided in this paper might be not enough to find the optimum value as the grid could be made more detail. However, this paper able to present the trend of the performance by varying $C$ and $\gamma$, opposing with which just informs the optimum value.

\section{Limitations}

The study proposes an AF detection employing SVM algorithm. SVM needs optimal parameters to find the best performance. This paper presents the performance with the SVM parameters $C$ of $1,10,100,1000,10,000$ and 100,000 and its kernel function parameter $\gamma$ of 1, 10, 100 and 1000. The more detail values, such as $C$ of 100, 200,300, are not tested. It may be that the values are the optimal one. However, considering the accuracy trend in Figure 6 we might have the optimal accuracy.

\section{Future works}

Implementation of the proposed AF detection approach in a real device is a challenge and is brought to the attention of the next works. The works are in line with studies to obtain sophisticated AF detection devices, such as by measuring performance of a Smartphonebased atrial fibrillation detection (Chang et al., 2015; Sardana et al., 2016). 


\section{Conclusion}

We have presented a strategy of atrial fibrillation (AF) detection. An SVM with electrocardiographic features have been tested using clinical data. The electrocardiographic features include the descriptive statistics of RR intervals in a segment or window. Different descriptive statistics have been tested and they could provide significant contribution for AF detection. SVM with different free parameters are tested. The optimal SVM parameters could provide a good performance of the AF detection. Using clinical data we have tested the approach and we find AF detection with the performance of $98.47 \%$ and $97.84 \%$, in terms of sensitivity and specificity. This AF detection approach is still need improvement to find a higher performance before it can be used for an AF diagnostic tool.

\section{References}

Asgari, S., Mehrnia, A. and Moussavi, M. (2015) 'Automatic detection of atrial fibrillation using stationary wavelet transform and support vector machine', Computers in Biology and Medicine, Vol. 60, pp.132-142.

Burges, C. (1998) 'A tutorial on support vector machines for pattern recognition', Data Mining and Knowledge Discovery, Vol. 2, No. 2, pp.121-167.

Chang, W-L., Hou, C.J-Y., Wei, S-P., Tsai, J-P., Chen, Y-J., Chen, M-L., Chuech, C-C., Hung, CL., Huang, M-Y. and Lee, C-H. (2015) 'Utilization and clinical feasibility of a handheld remote electrocardiography recording device in cardiac arrhythmias and atrial fibrillation: a pilot study', International Journal of Gerontology, Vol. 9, No. 4, pp.206-210.

Chugh, S.S., Havmoeller, R., Narayanan, K., Singh, D., Rienstra, M., Benjamin, E.J., Gillum, R.F., Kim, Y-H., McAnulty, J.H., Zheng, Z-J., Forouzanfar, M.H., Naghavi, M., Mensah, G.A., Ezzati, M. and Murray, C.J.L. (2014) 'Worldwide epidemiology of atrial fibrillation: a global burden of disease 2010 study', Circulation, Vol. 129, No. 8, pp.837-847.

Colloca, R., Johnson, A.E., Mainardi, L. and Clifford, G.D. (2013) 'A support vector machine approach for reliable detection of atrial fibrillation events', Computing in Cardiology Conference (CinC), Zaragoza, Spain, pp.1047-1050.

Daqrouq, K., Alkhateeb, A., Ajour, M.N. and Morfeq, A. (2014) 'Neural network and wavelet average framing percentage energy for atrial fibrillation classification', Computer Methods and Programs in Biomedicine, Vol. 113, No. 3, pp.919-926.

Foley, A.M., Leahy, P.G., Marvuglia, A. and McKeogh, E.J. (2012) 'Current methods and advances in forecasting of wind power generation', Renewable Energy, Vol. 37, No. 1, pp.1-8.

Goldberger, A.L., Amaral, L.A.N., Glass, L., Hausdorff, J.M., Ivanov, P.C., Mark, R.G., Mietus, J.E., Moody, G.B., Peng, C.K. and Stanley, H.E. (2000) 'PhysioBank, PhysioToolkit, and PhysioNet: components of a new research resource for complex physiologic signals', Circulation, Vol. 101, No. 23, pp.e215-e220.

Healey, J.S., Connolly, S.J., Gold, M.R., Israel, C.W., Van Gelder, I.C., Capucci, A., Lau, C., Fain, E., Yang, S. and Bailleul, C. (2012) 'Subclinical atrial fibrillation and the risk of stroke', New England Journal of Medicine, Vol. 366, No. 2, pp.120-129.

Huang, C., Ye, S., Chen, H., Li, D., He, F. and Tu, Y. (2011) 'A novel method for detection of the transition between atrial fibrillation and sinus rhythm', IEEE Transactions on Biomedical Engineering, Vol. 58, No. 4, pp.1113-1119.

Jiang, K., Huang, C., Ye, S-M. and Chen, H. (2012) 'High accuracy in automatic detection of atrial fibrillation for Holter monitoring', Journal of Zhejiang University Science B, Vol. 13, No. 9, pp.751-756. 
Lainscak, M., Dagres, N., Filippatos, G.S., Anker, S.D. and Kremastinos, D.T. (2008) 'Atrial fibrillation in chronic non-cardiac disease: where do we stand?', International Journal of Cardiology, Vol. 128, No. 3, pp.311-315.

Larburu, N., Lopetegi, T. and Romero, I. (2011) 'Comparative study of algorithms for atrial fibrillation detection', Computing in Cardiology, Vol. 2011, pp.265-268.

Lee, J., Nam, Y., McManus, D.D. and Chon, K.H. (2013) 'Time-varying coherence function for atrial fibrillation detection', IEEE Transactions on Biomedical Engineering, Vol. 60, No. 10, pp.2783-2793.

Li, H., Chung, F-l. and Wang, S. (2015) 'A SVM based classification method for homogeneous data', Applied Soft Computing, Vol. 36, pp.228-235.

Lian, J., Wang, L. and Muessig, D. (2011) 'A simple method to detect atrial fibrillation using RR intervals', The American Journal of Cardiology, Vol. 107, No. 10, pp.1494-1497.

Mateo, J. and Rieta, J.J. (2013) 'Radial basis function neural networks applied to efficient QRST cancellation in atrial fibrillation', Computers in Biology and Medicine, Vol. 43, No. 2, pp.154-163.

Mohebbi, M. and Ghassemian, H. (2008) 'Detection of atrial fibrillation episodes using SVM', Engineering in Medicine and Biology Society, 2008. EMBS 2008. 30th Annual International Conference of the IEEE, 20-25 August, Vancouver, British Columbia, pp.177-180.

Nuryani, N., Ling, S. and Nguyen, H. (2012) 'Electrocardiographic signals and swarm-based support vector machine for hypoglycemia detection', Annals of Biomedical Engineering, Vol. 40, pp.934-945.

Nuryani, N., Yahya, I. and Lestari, A. (2014) 'Premature ventricular contraction detection using swarm-based support vector machine and QRS wave features', International Journal of Biomedical Engineering and Technology, Vol. 16, No. 4, pp.306-316.

Preetha, R. and Suresh, G. (2014) 'Performance evaluation of immune-inspired support vector machine', International Journal of Biomedical Engineering and Technology, Vol. 16, No. 3, pp.209-222.

Saini, I., Singh, D. and Khosla, A. (2014) 'Electrocardiogram beat classification using empirical mode decomposition and multiclass directed acyclic graph support vector machine', Computers \& Electrical Engineering, Vol. 40, No. 5, pp.1774-1787.

Saleh, M.R., Martín-Valdivia, M.T., Montejo-Ráez, A. and Ureña-López, L. (2011) 'Experiments with SVM to classify opinions in different domains', Expert Systems with Applications, Vol. 38, No. 12, pp.14799-14804.

Sardana, M., Saczynski, J., Esa, N., Floyd, K., Chon, K., Chong, J.W. and McManus, D. (2016) 'Performance and usability of a novel Smartphone application for atrial fibrillation detection in an ambulatory population referred for cardiac monitoring', Journal of the American College of Cardiology, Vol. 67, No. 13_S, pp.844-844.

Suchetha, M. and Kumaravel, N. (2013) 'Classification of arrhythmia in electrocardiogram using EMD based features and support vector machine with margin sampling', International Journal of Computational Intelligence and Applications, Vol. 12, No. 3, Article no. 1350015. 